\title{
Caractérisation de la diversité phénotypique et génotypique du Sorgho pluvial dans la zone soudano sahélienne du Cameroun.
}

\author{
DANBE Nicodème ${ }^{1 *}$, YAKOUBA Oumarou ${ }^{2}$, SOBDA Gonné1, BASGA Simon DJAKBA ${ }^{1}$, LENDZEMO \\ Venasius ${ }^{1}$, KAOUVON Philémon ${ }^{1}$, DICKMI VAILAM Claudette ${ }^{1}$, SUH Christopher ${ }^{1}$, DJONNEWA André1, \\ YOURI Alphonse ${ }^{1}$, KABOUI André ${ }^{3}$ \\ ${ }_{1}^{1} \mathrm{RAD}$ : Institut de Recherche Agricole pour le Développement \\ 2ENSPM-UMa : École Nationale Supérieure Polytechnique de Maroua-Université de Maroua (Département d'Agriculture, \\ Élevage et Produits Dérivés). \\ ${ }^{3}$ ONG SAHELI \\ *Auteur correspondant, E-mail : danbenico@yahoo.fr Téléphone : (+237) 694061076
}

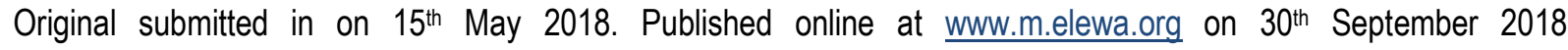
http://dx.doi.org/10.4314/jab.v129i1.1

\section{RÉSUMÉ}

Objectif : Cette étude a pour objectif d'évaluer la diversité génotypique et phénotypique existante au sein des accessions de Sorgho pluvial de la zone soudano sahélienne du Cameroun et d'estimer les paramètres génétiques permettant d'orienter les futurs programmes d'amélioration variétale.

Méthodologie et résultats: L'étude réalisée sur 44 accessions selon un dispositif en blocs de Fisher complètement randomisé à trois répétitions révèle une grande diversité morphologique organisée autour des caractères végétatifs et de rendement avec un groupement des accessions en trois dont le troisième renferme les accessions performantes en termes de hauteur de tiges et rendement grains. Une faible influence des facteurs environnementaux sur l'expression des performances des accessions a été observée avec des faibles écarts entre les coefficients de variation phénotypique et génotypique, une forte héritabilité au sens large pour tous les caractères.

Conclusion et application : En somme, Tous les caractères étudiés ont exprimé une forte héritabilité au sens large avec un gain génétique attendu variant, mais fort chez les caractères végétatifs et modéré chez les caractères liés au rendement. La grande diversité génétique observée au sein des accessions de sorgho étudiées montre que des possibilités de sélection existent. En outre les accessions du groupe trois constituent des potentiels parents pouvant servir dans les programmes d'amélioration ou de création variétale au Cameroun.

Mots clés : Sorgho pluvial, zone soudano sahélienne du Cameroun, diversité génotypique et phénotypique. 


\section{ABSTRACT}

Assessment of genotypic and phenotypic diversity of the rain-fed Sorghum in the sahelian zone of Cameroon. Objectives: This study was conducted to determine the genotypic and phenotypic diversity in the accession of the rain-fed Sorghum in the sahelian zone of Cameroon and to estimate the genetic parameters, which can be used to create new varieties of Sorghum in Cameroon.

Methodology and results: Forty-four (44) accessions were grown in a randomized complete bloc design with three replications. The results showed there was a large morphological diversity among the Sorghum accessions. Furthermore, these diversities were organized around vegetative and yield characters, which divided the accessions into three groups. The individual in group three (3) showed interesting performance in term of plant height and grain yield parameters. The analysis of genotypic and phenotypic parameters showed that there was low influence of the environmental factors on the performances of the accessions.

Conclusions applications of findings : There was a the low difference between the phenotypic and genotypic variances for all characters. High broad sense heritability estimates recorded for all characters and the expected genetic advance were variables. The high expected genetic advance observed in the vegetative characters are better in the yield characters. The results from this study provide useful information in selection of parents that breeders could exploit in sorghum improvement program in Cameroon.

Keywords: raining season Sorghum, Sahelian zone of Cameroon, genotypic and phenotypic variances.

\section{INTRODUCTION}

Principale alimentation de la population de la zone soudano sahélienne du Cameroun, le Sorgho [Sorghum bicolor (L.) Moench] est la céréale la plus cultivée avec une superficie moyenne de 0,8 millions d'ha et une production moyenne de 1,15 millions de tonnes (USDA, 2016). II faut noter que deux types de sorgho sont cultivés au Nord Cameroun : les sorghos de saison des pluies et les sorghos de saison sèche (Kenga et Djorowé, 2008). Le sorgho de saison sèche, communément appelés « Muskwari ", est repiqué sur des vertisols alors que le Sorgho pluvial est semé dès le début de la saison pluvieuse et il termine son cycle en fin de saison des pluies. Tous les travaux portant sur la génétique du sorgho dans le passé s'étaient concentrés sur l'amélioration des variétés cultivées. Ils ont permis la mise au point des variétés de Sorgho tolérantes au Striga, à la sècheresse et des variétés précoces. Cependant,

\section{MATÉRIELS ET MÉTHODES}

Site d'expérimentation : Cette étude est menée en 2015 à la ferme expérimentale de l'Institut de Recherche Agricole pour le Développement (IRAD) de Maroua située à environ $10 \mathrm{Km}$ sur l'axe Maroua-Bogo à $14.31081^{\circ}$ et de longitude Ouest $10.59471^{\circ}$ de latitude Nord. Pendant cette étude, la pluviométrie moyenne de la zone était de $708 \mathrm{~mm}$ et 50 jours de pluie. ces variétés subissent les effets des perturbations climatiques telles que poches de sècheresses, le retard, l'insuffisance ou l'arrêt précoce des pluies et les inondations, (Yakouba et al., 2017), contribuant ainsi à la diminution continuelle des rendements. Une étude d'évaluation génétique des écotypes du Sorgho pluvial est donc nécessaire car elle constitue une base permettant la création des nouvelles variétés possédant des caractères souhaités. Certains travaux d'inventaire et de classification ont été effectués dans le passé, mais ils portaient sur le Sorgho repiqué (Kenga et Djorowé, 2008). La présente étude a pour objectif d'évaluer la diversité agromorphologique du Sorgho pluvial du Nord Cameroun et d'estimer les paramètres génétiques afin d'identifier les meilleures stratégies de valorisation de cette ressource génétique.

Matériel végétal: le matériel était constitué de 44 accessions de Sorgho pluvial de la descendance (S1) obtenus par autofécondation en 2014. La collection est issue des six départements de la Région de l'ExtrêmeNord Cameroun (Figure1). 


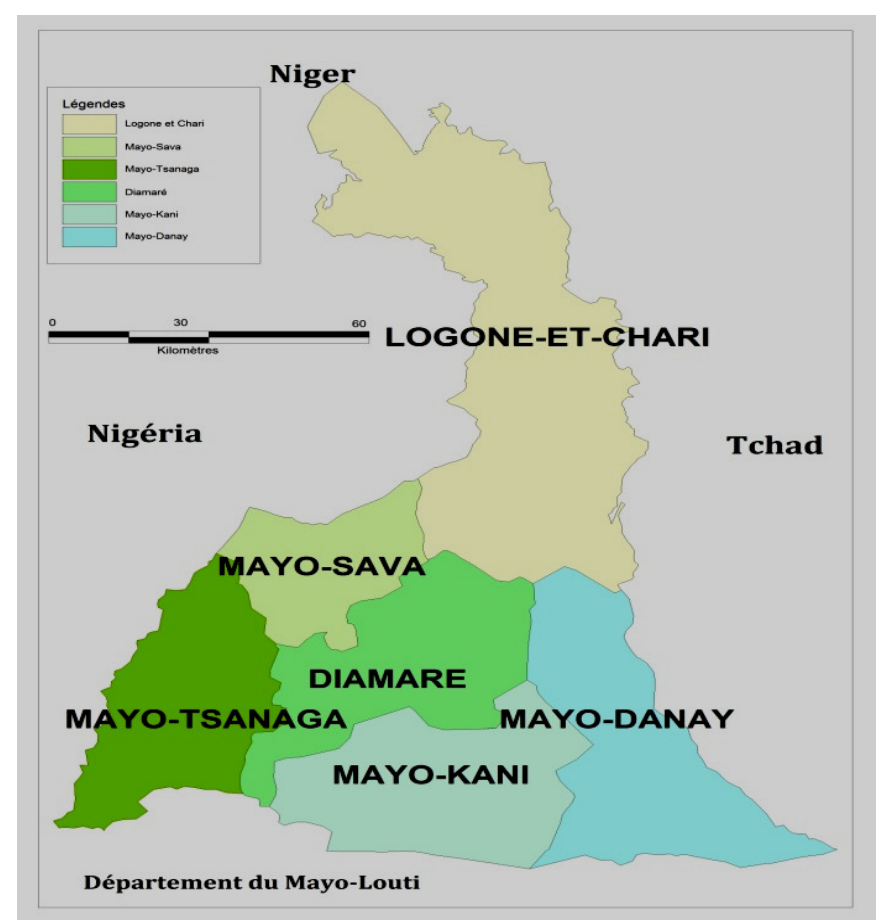

Figure 1: Localisation de la zone de collecte des accessions.

Méthode : Dispositif expérimental : L'étude a été menée suivant un dispositif en blocs complètement randomisés avec 3 répétitions. Chaque parcelle élémentaire était constituée de 2 lignes de $4 \mathrm{~m}$ de long. Les écartements étaient de $0.8 \mathrm{~m}$ entre les lignes et $0.40 \mathrm{~m}$ entre les poquets soit un total de 10 poquets par ligne. A deux semaines après levée, le démariage s'est fait pour laisser 2 plants par poquet. Un épandage d'engrais de fond NPK à la dose de $100 \mathrm{~kg} / \mathrm{ha}$ et d'urée $(50 \mathrm{~kg} / \mathrm{ha})$ a été respectivement apporté au labour et au stade gonflement de la panicule.

Collecte des données: Huit (09) caractères ont été observés dans cette étude. II s'agit de la durée du cycle semis $50 \%$ épiaison (jours), la hauteur de la tige principale $(\mathrm{cm})$, le nombre de feuilles de la tige principale (dénombré hebdomadairement à partir du stade 4-5 feuilles), la longueur de la troisième feuille sous paniculaire a été mesurée $(\mathrm{cm})$, la largeur de la troisième feuille sous paniculaire a été mesurée $(\mathrm{cm})$, la longueur de la panicule a été mesurée $(\mathrm{cm})$, le poids moyen de panicules ( 5 plantes/parcelle) a été pesé $(\mathrm{g})$, le poids moyen de grains ( 5 plantes/parcelle) a été pesé $(\mathrm{g})$, le poids de 1000 grains a été pesé $(\mathrm{g})$.

Analyse des données: Les analyses de variance ont été faites avec le logiciel Genstat (BreedingView) Release 18.0. Les paramètres génétiques ont été estimés à partir des composantes de l'analyse de variance. Les paramètres génétiques estimés ont permis la réalisation du dendrogramme (classification ascendante) avec le Logiciel $R$ 3.4.0. Les variances génotypique et phénotypique (VG et VP), les coefficients de variation génotypique et phénotypique (GCV et PCV), I'héritabilité au sens large $\left(\mathrm{H}^{2}\right)$ et le gain génétique attendu (GA) ont été calculés selon les formules utilisées par (Johnson et al., 1955) ; (Assefa et al., 2001) ; (Rex, 2002) ; (Hosseini et al., 2012) ; (Sawadogo et al., 2014) présentées dans le tableau 1. 

pluvial dans la zone soudano sahélienne du Cameroun

Tableau 1: Formules des différents paramètres génétiques estimés

\begin{tabular}{|c|c|c|}
\hline Paramètres & Formules & Signification des termes \\
\hline $\begin{array}{l}\text { Variance génotypique (VG) } \\
\text { Variance phénotypique (VP) } \\
\text { Héritabilité au sens large }\end{array}$ & $\begin{array}{l}V G=(M S G-M S E) / r \\
V P=V G+(M S E / r)=M S G / r \\
H^{2}(\%)=(V G / V P)^{*} 100\end{array}$ & $\begin{array}{l}\text { MSG : carré moyen des génotypes } \\
\text { MSE : carré moyen de l'erreur } \\
r: \text { nombre de répétitions }\end{array}$ \\
\hline $\begin{array}{l}\text { Coefficient de variation génotypique (GCV) } \\
\text { Coefficient de variation phénotypique } \\
\text { (PCV) } \\
\text { Gain génétique attendu (GA) } \\
\text { Gain génétique attendu par rapport à la } \\
\text { moyenne [GA (\% moyenne caractère)] }\end{array}$ & $\begin{array}{l}\mathrm{GCV}(\%)=(\sqrt{\mathrm{VG}} / \mathrm{X})^{*} 100 \\
\mathrm{PCV}(\%)=(\sqrt{\mathrm{VP}} / \mathrm{X})^{*} 100 \\
\mathrm{GA}=\mathrm{H}^{2 *} \sqrt{\mathrm{VP}}{ }^{*} \mid \\
\mathrm{GA}(\% \text { moyenne caractère }) \\
= \\
(\mathrm{GA} X){ }^{*} 100\end{array}$ & $\begin{array}{l}\sqrt{V G}: \text { écart-type de la variance génotypique } \\
\sqrt{V P}: \text { écart-type de la variance } \\
\text { phénotypique } \\
\text { I: constante. Avec un coefficient } \\
\text { de sélection de } 5 \% \text {, est } 2,06 \\
\mathrm{X}: \text { moyenne du caractère } \\
\mathrm{H}^{2}: \text { Héritabilité au sens large } \\
\mathrm{GA}: \text { gain génétique attendu }\end{array}$ \\
\hline
\end{tabular}

\section{RÉSULTATS}

Variation des caractères agromorphologiques: Le Tableau 2 montre que tous les caractères discriminent les accessions du sorgho pluvial de la zone soudano sahélienne du Cameroun à l'exception de la longueur de la troisième feuille sous paniculaire (LOF). II s'agit des caractères nombre de jours pour obtenir $50 \%$ d'épiaison (NJF), la hauteur de la tige principale (HT), le nombre de feuilles de la tige principale (NF), la largeur de la troisième feuille sous paniculaire (LAF), la longueur de la panicule (LP), le Rendement grains (Rdt. grs), le poids de 1000 grains (PMG), la vigueur de la plante (VP). Les caractères (VP, HT, Rdt.grs, PMG) ayant des coefficients de variation $<30 \%$ varient énormément. Ces accessions fleurissent entre 54 et 96 jours après semis et ont des panicules longues allant de 14,8 à $52,2 \mathrm{~cm}$, une hauteur moyenne de $224,01 \mathrm{~cm}$, un rendement moyen grain par plant de $110,87 \mathrm{~g}$, et un poids moyen de mille grains de $21,89 \mathrm{~g}$.

Tableau 2: Analyse de variance des 9 caractères étudiés.

\begin{tabular}{l|l|l|l|l|l|l}
\hline Caractères & Moy & Min & Max & MSG & MSE & CV (\%) \\
\hline VP & $3,07 \pm 0,95$ & 1 & 5 & $11,63^{*}$ & 0,17 & 31 \\
NJF (jrs) & $73,15 \pm 11,27$ & 54 & 96 & $56,77^{\star \star \star}$ & 1,44 & 15 \\
NF & $10,30 \pm 1,04$ & 7 & 13 & $21,25^{\star \star \star}$ & 0,21 & 10 \\
LAF $(\mathrm{cm})$ & $7,16 \pm 1,04$ & 5,18 & 9,88 & $17,75^{\star \star}$ & 0,16 & 15 \\
LOF $(\mathrm{cm})$ & $71,08 \pm 8,66$ & 54,64 & 86,82 & $55,95 \mathrm{~ns}$ & 0,88 & 12 \\
HT $(\mathrm{cm})$ & $224,01 \pm 75,19$ & 60 & 467,2 & $99,39^{\star \star \star}$ & 8,71 & 34 \\
LP $(\mathrm{cm})$ & $30,70 \pm 7,86$ & 14,8 & 52,2 & $36,73^{\star *}$ & 0,85 & 26 \\
Rdt.grs $(\mathrm{g})$ & $110,87 \pm 142,72$ & 5 & 141,6 & $65,03^{\star * \star}$ & 17,5 & 129 \\
PMG $(\mathrm{g})$ & $21,89 \pm 8,08$ & 11 & 28,9 & $28,16^{*}$ & 1,24 & 37 \\
\hline
\end{tabular}

VP: Vigueur des plantes, NJF : Nombre de Jours de Floraison, NF : Nombre de Feuilles, LAF : Largeur de la troisième Feuille sous paniculaire, LOF : Longueur de la troisième Feuille sous paniculaire, HT: Hauteur des Plantes, LP : Longueur des Panicules, Rdt.grs : Rendement Grains par plantes, PMG : Poids de Mille Grains, Moy : Moyenne des caractères, Min : Minimum, Max : Maximum, MSG : Carré Moyenne des Génotypes, MSE : Carré moyenne des Erreurs, CV : Coefficient de Variation, * : différence significative à $5 \%,{ }^{* *}$ : différence hautement significative à $1 \%,{ }^{* * *}$ : différence très significative à $1 \%$, ns : Non significatif.

Diversité morphologique des accessions du Sorgho de la zone soudano sahélienne du Cameroun: L'analyse en composante principale (Tableau 3) révèle que les trois premières composantes avec les neuf paramètres mesurés expliquent $73,071 \%$ de la variabilité totale (Tableau 3) dont 40,104\% pour l'axe 1, 20,490\% pour l'axe 2, et $12,477 \%$ pour l'axe 3 . La variabilité globale est expliquée par neuf composantes principales avec des valeurs propres comprises entre 1,123 et 3,609. Le premier axe est défini par les caractères ayant trait au rendement (Rdt.grs, $P M G)$, à la tige $(H T)$, à la feuille (NF), et à la floraison (NJF). La deuxième composante qui est corrélée avec la largeur et la longueur des feuilles (LAF, LOF) est celle du bon développement végétatif. Le 
troisième axe est corrélé positivement avec la vigueur des panicules (LP).

plantes (VP) et négativement avec la longueur des

Tableau 3: Valeurs propres et représentation de la variation par les 3 premières composantes principales (ACP) à partir de 9 caractères

\begin{tabular}{l|l|l|l}
\hline Composante principale & Groupe.1 & Groupe.2 & Groupe.3 \\
\hline Variance & 3,609 & 1,844 & 1,123 \\
$\%$ of var. & 40,104 & 20,490 & 12,477 \\
Cumulative \% of var. & 40,104 & 60,594 & 73,071 \\
\hline VP & $-0,201$ & $-0,387$ & $0,744^{*}$ \\
NJF (jrs) & $0,866^{*}$ & $-0,187$ & 0,170 \\
NF & $0,685^{*}$ & 0,279 & 0,245 \\
LAF $(\mathrm{cm})$ & 0,312 & $0,868^{*}$ & 0,075 \\
LOF $(\mathrm{cm})$ & 0,490 & $0,641^{*}$ & $-0,091$ \\
HT $(\mathrm{cm})$ & $0,874^{*}$ & $-0,060$ & $-0,073$ \\
LP $(\mathrm{cm})$ & 0,347 & $-0,456$ & $-0,661^{*}$ \\
Rdt.grs $(\mathrm{g})$ & $0,747^{*}$ & $-0,344$ & 0,047 \\
PMG $(\mathrm{g})$ & $0,756^{*}$ & $-0,298$ & 0,151 \\
\hline VP:Vign
\end{tabular}

VP : Vigueur des plantes, NJF : Nombre de Jours de Floraison, NF : Nombre de Feuilles, LAF : Largeur de la troisième Feuille sous paniculaire, LOF : Longueur de la troisième Feuille sous paniculaire, HT: Hauteur des Plantes, LP : Longueur des Panicules, Rdt.grs : Rendement Grains par plantes, PMG : Poids de Mille Grains, * : différence significative à $5 \%$.

Analyse de la diversité génotypique : La classification ascendante hiérarchique (Figure 2) donne une structuration des 44 accessions de Sorgho pluvial de la zone soudano sahélienne du Cameroun en 3 groupes. Les trois groupes ainsi obtenus renferment respectivement 3 accessions pour le premier groupe, 31 accessions pour le deuxième et 10 pour le troisième groupe. Les résultats des analyses de variance dans le tableau 4 montrent une différence hautement significative au seuil de $1 \%$ pour les caractères végétatifs nombre de feuilles (NF), largeur des feuilles (LAF), longueur des feuilles (LOF), la hauteur des tiges (HT). Ainsi donc, les accessions du groupe 3 présentent tous les caractères au dessus des moyennes générales excepté le caractère (VP : 3,03). Le groupe 2 regroupe les accessions ayant des caractères qui ont de performance moins que la moyenne de performance générale excepté la largeur des feuilles qui est légèrement supérieur (LAF :7,25cm). Le groupe 1 est un groupe qui renferme des accessions qui présentent les caractères qui sont inférieurs aux caractères des deux autres groupes à l'exception $\mathrm{du}$ caractère (VP : 3,33).

Tableau 4: Performances moyennes de Sorgho pluvial de la zone soudano sahélienne du Cameroun.

\begin{tabular}{l|lll|llll}
\hline \multirow{2}{*}{ Caractères } & Collection & & \multicolumn{5}{l}{ Groupes } \\
\cline { 2 - 9 } & Moy & Min & Max & $\mathbf{1}$ & $\mathbf{2}$ & $\mathbf{3}$ & Pr \\
\hline VP & $3,07 \pm 0,95$ & 1 & 5 & 3,33 & 3,06 & 3,03 & 0,711 \\
NJF (jrs) & $73,15 \pm 11,27$ & 54 & 96 & 64,33 & 68,29 & 85,93 & $1,54 \mathrm{e}-13^{* * *}$ \\
NF & $10,30 \pm 1,04$ & 7 & 13 & 8,38 & 10,23 & 10,74 & $7,26 \mathrm{e}-07^{* * *}$ \\
LAF $(\mathrm{cm})$ & $7,16 \pm 1,04$ & 5,18 & 9,88 & 5,62 & 7,25 & 7,30 & $0,000304^{* * *}$ \\
LOF $(\mathrm{cm})$ & $71,08 \pm 8,66$ & 54,64 & 86,82 & 60,81 & 70,72 & 74,43 & $5,6 \mathrm{e}-05^{* * *}$ \\
HT $(\mathrm{cm})$ & $224,01 \pm 75,19$ & 60 & 467,2 & 134,62 & 209,29 & 278,00 & $1,81 \mathrm{e}-06^{* * *}$ \\
LP $(\mathrm{cm})$ & $30,70 \pm 7,86$ & 14,8 & 52,2 & 30,2 & 29,76 & 32,72 & 0,289 \\
Rdt.grs $(\mathrm{g})$ & $110,87 \pm 142,72$ & 5 & 141,6 & 35,82 & 31,81 & 248,47 & $9,59 \mathrm{e}-12^{* * *}$ \\
PMG $(\mathrm{g})$ & $21,89 \pm 8,08$ & 11 & 28,9 & 15,20 & 16,12 & 25,15 & $0,000327^{\star * *}$ \\
Nombre d'accessions & 44 & - & - & 3 & 31 & 10 & - \\
\hline
\end{tabular}

VP : Vigueur des plantes, NJF : Nombre de Jours de Floraison, NF : Nombre de Feuilles, LAF : Largeur de la troisième Feuille sous paniculaire, LOF : Longueur de la troisième Feuille sous paniculaire, HT : Hauteur des Plantes, LP : Longueur des Panicules, Rdt.grs : Rendement Grains par plantes, PMG : Poids de Mille Grains, Moy : Moyenne des caractères, Min : Minimum, Max: Maximum, ${ }^{* * *}$ : différence très significative à $1 \%$, Pr: Probabilité $F$. 


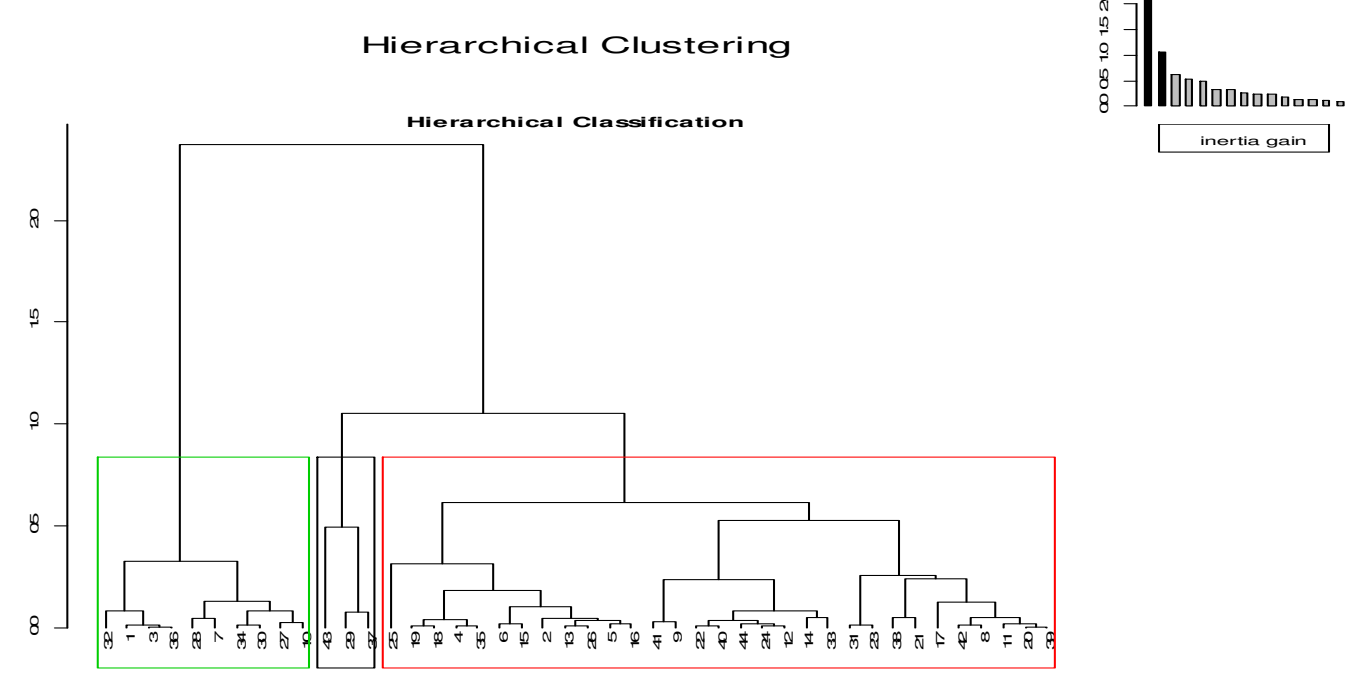

Figure 2 : Classification ascendante de 44 accessions de Sorgho pluvial de la zone soudano sahélienne du Cameroun.

\section{Estimation des paramètres génétiques}

Variance phénotypique et génotypique: Les paramètres génétiques montrent que la variance phénotypique est supérieure à la variance génotypique pour tous les paramètres (du tableau 5). La variance phénotypique varie de 3,88 à 33,13 alors que la variance génotypique varie de 3,82 à 30,23 . Le caractère Hauteur de la tige $(33,13)$ présente la variance phénotypique la plus élevée suivi du rendement grains par plante $(21,68)$. La longueur des feuilles $(18,65 \mathrm{~cm})$, le nombre de jours de floraison après semis $(18,92)$ et la longueur des panicules $(12,24 \mathrm{~cm})$ présentent des variances phénotypiques modérées (<20). A l'exception de la hauteur de tige qui présente une variance génotypique élevée, tous les autres caractères ont des variances génotypiques soit modérées ou faibles $(<10)$ tels que (Vigueur des Plantes, Nombre des Feuilles, Largeur des Feuilles, et Poids de Mille Grains).

Coefficients de variation phénotypique et génotypique : Pour tous les caractères, le coefficient de variation phénotypique est plus élevé que le coefficient de variation génotypique (Tableau 5). C'est le caractère vigueur des plantes qui a les coefficients de variation phénotypique et génotypique les plus élevés respectivement 64,13 et $63,66 \%$. II est suivi des caractères foliaires notamment largeur de feuilles (LAF : 33,97 et $33,82 \%$ ) et le nombre de feuilles (NF : 25,84 et $25,71 \%)$. Les caractères ayant des coefficients de variation phénotypique et génotypiques modérés sont le poids de mille grains (PMG) et la longueur de panicules (LP). Les coefficients de variation phénotypique et génotypique faibles sont observés pour les caractères rendement grains (Rdt.grs), hauteur de tige (HT), longueur de feuilles (LOF) et nombre de jours de floraison après semis (NJF).

Héritabilité au sens large: Le Tableau 5 présente l'héritabilité des caractères ainsi qu'ils suivent : Largeur des Feuilles : 99,10\% ; Nombre des Feuilles : 99,01\% ; Vigueur des Plantes : 98,54\% ; Longueur des Feuilles: 98,43\% ; Longueur des Panicules : $97,69 \%$; Nombre des Jours 50\% Floraison : $97,46 \%$; Poids de Mille Grains : 95,60\% ; et Hauteur des Plantes : $91,24 \%$, Rendement grains: $73,09 \%$.

Gain génétique attendu : Le gain génétique attendu par rapport à la moyenne du caractère GA (\% moy caract.) a varié énormément en fonction des caractères allant de 4,83 à 130,19\% (Tableau 5). Les caractères ayant de faibles gains attendus sont (Hauteur des plants, Rendement grains). Les caractères ayant les gains attendus modérés sont (Nombre de Jours pour obtenir 50 $\%$ d'épiaison, Longueur de la troisième feuille sous paniculaire) et ceux qui ont les gains attendus moyens sont (Poids Mille Grains, Longueur des Panicules). Les caractères ayant des gains génétiques attendus plus élevés sont (Nombre des Feuilles, Largeur des Feuilles, Vigueur des Plants). 

pluvial dans la zone soudano sahélienne du Cameroun

Tableau 5: Paramètres génétiques calculés du Sorgho pluvial de la zone soudano sahélienne du Cameroun.

\begin{tabular}{|c|c|c|c|c|c|c|c|c|c|c|}
\hline & VG & VP & $\mathrm{H}^{2}(\%)$ & $\sqrt{V G}$ & $\sqrt{V P}$ & $\mathrm{X}$ & GCV (\%) & PCV(\%) & GA & $\begin{array}{c}\mathrm{GA}(\% \text { moy } \\
\text { caract.) }\end{array}$ \\
\hline VP & 3,82 & 3,88 & 98,54 & 1,95 & 1,97 & 3,07 & 63,66 & 64,13 & 4,00 & 130,19 \\
\hline NJF (jrs) & 18,44 & 18,92 & 97,46 & 4,29 & 4,35 & 73,15 & 5,87 & 5,95 & 8,73 & 11,94 \\
\hline NF & 7,01 & 7,08 & 99,01 & 2,65 & 2,66 & 10,30 & 25,71 & 25,84 & 5,43 & 52,70 \\
\hline LAF (cm) & 5,86 & 5,92 & 99,10 & 2,42 & 2,43 & 7,16 & 33,82 & 33,97 & 4,97 & 69,35 \\
\hline $\operatorname{LOF}(\mathrm{cm})$ & 18,36 & 18,65 & 98,43 & 4,28 & 4,32 & 71,08 & 6,03 & 6,08 & 8,76 & 12,32 \\
\hline $\mathrm{HT}$ (cm) & 30,23 & 33,13 & 91,24 & 5,50 & 5,76 & 224,01 & 2,45 & 2,57 & 10,82 & 4,83 \\
\hline $\mathrm{LP}(\mathrm{cm})$ & 11,96 & 12,24 & 97,69 & 3,46 & 3,50 & 30,70 & 11,26 & 11,40 & 7,04 & 22,94 \\
\hline Rdt.grs (g) & 15,84 & 21,68 & 73,09 & 3,98 & 4,66 & 110,87 & 3,59 & 4,20 & 7,01 & 6,32 \\
\hline PMG (g) & 8,97 & 9,39 & 95,60 & 3,00 & 3,06 & 21,89 & 13,68 & 14,00 & 6,03 & 27,56 \\
\hline
\end{tabular}

VP: Vigueur des plantes, NJF : Nombre de Jours de $50 \%$ Floraison, NF : Nombre de Feuilles, LAF : Largeur de la troisième Feuille sous paniculaire, LOF : Longueur de la troisième Feuille sous paniculaire, HT : Hauteur des Plantes, LP : Longueur des Panicules, Rdt.grs : Rendement Grains par plantes, PMG : Poids de Mille Grains, X: Moyenne des caractères, VG : Variance Génotypique, VP : Variance Phénotypique, $H^{2}$ : Héritabilité au sens large, $\sqrt{\boldsymbol{V G}}$ : Ecartype de la variance génotypique, $\sqrt{\boldsymbol{V P}}$ : Ecartype de la variance Phénotypique, GCV : Coefficient de Variation Génotypique, PCV : Coefficient de Variation Phénotypique, GA : Gain génétique Attendu.

\section{DISCUSSION}

Les coefficients de variation qui sont élevés et l'écart important entre les valeurs minimales et maximales des caractères traduisent la variabilité qui existe dans la collection des accessions étudiées. Ces résultats corroborent les travaux menés par (Ayana et Bekele, 2000) sur les accessions de sorghos de l'Éthiopie et de l'Érythrée et ceux de (Nebié et al., 2012) sur les sorghos à grains sucrés au Centre-nord du Burkina. Cette variabilité est justifiable par le fait que les populations s'échangent des semences lors de la période de semis et elles font elles-mêmes la sélection des semences sans utiliser des critères de différenciation suffisamment rigoureux. Cela favorise donc le flux des gènes qui contribuent à la variabilité génétique. Des observations similaires avaient été faites par (Nebié et al., 2012) et (Nerbéwendé et al., 2014). Les accessions de la zone soudano-sahélienne du Cameroun fleurissent entre 54 et 96 jours après semis et ont des panicules longues allant de 14,8 à $52,2 \mathrm{~cm}$, une hauteur moyenne de $224,01 \mathrm{~cm}$, un rendement moyen grain par plant de $110,87 \mathrm{~g}$, et un poids moyen de mille grains de $21,89 \mathrm{~g}$. Les études conduites par (Kenga et Djorowé, 2008) sur la variabilité morphologique du Sorgho de contre saison du Nord Cameroun ont révélé des écarts importants qui existent entre les minima et les maxima pour tous les caractères et aussi de grands coefficients de variation qui dépassent $30 \%$ pour les caractères hauteur de plant et masse de grain par panicule. La distribution du cycle semis nombre de jours à $50 \%$ de floraison varie entre les valeurs extrêmes de 25 jours et 86 jours. La hauteur des écotypes présente une moyenne de $180 \mathrm{~cm}$ pour des valeurs se situant dans l'intervalle de $125 \mathrm{~cm}$ pour les variétés de courte taille et $530 \mathrm{~cm}$ pour les variétés de grande taille. Les rendements en grain sont également très variables, avec une moyenne de $1,4 \mathrm{~kg} / \mathrm{ha}$, les maxima se situant autour de $2,5 \mathrm{~kg} / \mathrm{ha}$. La diversité morphologique sur la base des neuf caractères (Rdt.grs, PMG, HT, NF, NJF, LAF, LOF, VP, LP) en Analyse en Composante Principale (ACP) a révélé une très forte contribution de ces caractères à l'organisation de la diversité au sein du Sorgho pluvial au Cameroun. Cette structuration se justifierait par la manière de sélectionner les semences par les paysans qui fixent leurs caractères principaux sur le comportement phénotypique tel que la biomasse, la précocité, et le rendement. (Nerbéwendé et al., 2014) ; (Djè et al., 2007) ; (Koffi et al., 2011) avaient également fait ces mêmes constats respectivement sur des variétés traditionnelles de sorghos cultivés au Nord du Burkina-Faso, au Nord-ouest du Maroc et sur les sorghos cultivés au Nord de la Côte d'Ivoire. Les trois premières composantes avec les neuf paramètres mesurés expliquent $73.071 \%$ de la variabilité totale. (Kenga et Djorowé, 2008) ont fait ce même constat sur la variabilité au sein du Sorgho de contre saison au Nord Cameroun. Ils ont remarqué que les trois premières composantes représentaient à elles seules les $74 \%$ de la variabilité. Les différents groupes morphologiques obtenus dans cette étude traduisent la diversité morphologique qui existe entre les différentes accessions de Sorgho de la zone soudano sahélienne et cela donne une opportunité aux créateurs des variétés de Sorgho, le choix des parents dans le but d'obtenir des nouvelles 

pluvial dans la zone soudano sahélienne du Cameroun

variétés répondant mieux aux besoins des populations. Ce choix pourrait être orienté vers les accessions du groupe 3 qui présentent les meilleurs rendements grains. Les paramètres génétiques estimés ont montré que la variance génotypique est inférieure à la variance phénotypique de tous les caractères étudiés. Ces résultats concordent avec ceux de (Nerbéwendé et al., 2014) sur le sorgho à tiges sucrées, (Drabo et al., 2013) sur le mil, (Hosseini et al., 2012) sur le riz et (Doss et al., 2012) sur le mûrier. Les écarts entre ces deux variances (Variances génotypique et phénotypique) sont assez faibles. Ceci a été aussi observé par (Nerbéwendé et al., 2014) sur le Sorgho à tiges sucrées du Nord du Burkina. Ce faible écart pour tous les caractères traduirait un faible impact des facteurs environnementaux sur l'expression des caractères. De tels résultats ont été obtenus par (Govindaraj et al., 2011) sur le mil, (Hosseini et al., 2012) et (Drabo et al., 2013) sur le riz, (Nerbéwendé et al., 2014) sur le sorgho à tiges sucrées. La sélection est d'autant plus efficace que l'héritabilité des caractères est élevée (Nerbéwendé et al. 2014). L'estimation du coefficient de variation génotypique (GCV) et de l'héritabilité $\left(h^{2}\right)$ fournit la meilleure information pour le choix des parents à hybrider en vue d'obtenir les caractères recherchés (Burton et Devane, 1953 cité par Nerbéwendé et al. 2014). Selon (Sumathi et al., 2010), les coefficients de variation génotypique et phénotypique sont faibles en deçà de $11 \%$, modérés entre 11 et $20 \%$ et élevé au-delà de $20 \%$. Les coefficients de variation phénotypique et génotypique sont élevés pour le caractère vigueur des plantes (VP : 64,13 et 63,66\%), pour la largeur de feuilles (LAF : 33,97 et $33,82 \%$ ) et pour le nombre de feuilles (NF : 25,84 et $25,71 \%$ ). Les caractères ayant des coefficients de variation

\section{CONCLUSION}

Cette étude a révélé une grande variabilité morphologique au sein des sorghos pluviaux de la zone soudano sahélienne du Cameroun groupée autour des caractères végétatifs et du rendement. Les 44 accessions s'organisent en 3 groupes morphologiques distincts. Cette organisation est basée sur la hauteur de tiges et le rendement grains par plante. Le groupe 3 présente des accessions performantes en termes de hauteur de tiges et rendement grains par plante. II a été noté qu'il existe une faible influence des facteurs environnementaux sur l'expression des performances des accessions illustrée

\section{BIBLIOGRAPHIE}

Assefa K., Tefera H., Merker A., Kefyalew T. and Hundera F., 2001. Variability, heritability and phénotypique et génotypiques modérés sont le poids de mille grains (PMG : 14,00 et 13,68\%) et longueur de panicules (LP : 11,40 et $11,26 \%$ ). Les caractères qui ont des coefficients de variation phénotypique et génotypique faibles sont le rendement grains (Rdt.grs : 4,20 et 3,59\%), la hauteur de tige (HT : 2,57 et 2,45\%), la longueur de feuilles (LOF : 6,08 et 6,03\%) et le nombre de jours de floraison après semis (NJF : 5,95 et $5,87 \%$ ). Ces résultats vont dans le sens que ceux obtenus par (Nerbéwendé et al., 2014 ; Drabo et al., 2013) sur le cycle 50\% floraison et la hauteur du mil et du Sorgho. Selon (Johnson, 1955; Stanfield, 1975), l'héritabilité est élevée au-delà de $50 \%$, faible en deçà de $20 \%$ et moyenne entre 20 et $50 \%$. Dans cette étude, l'héritabilité au sens large est élevée pour tous les caractères étudiés car elle varie de 73,09 à $99,10 \%$. Ces résultats rejoignent ceux de (Vetriventhan et Nirmalakumari, 2007) sur le mil, (Ali et al., 2012 ; Nebié, 2014 ; Nerbéwendé et al., 2014) sur le sorgho. L'héritabilité forte des caractères traduit la faible influence des facteurs environnementaux sur leur expression. Dans ce cas le phénotype exprime véritablement le génotype des accessions (Visscher et al., 2008). En outre, I'héritabilité à elle seule ne permet pas de prédire si la sélection apportera une amélioration substantielle (Nerbéwendé et al., 2014). Néanmoins, l'estimation conjointe de l'héritabilité et du gain génétique attendu [GA (\% moyenne du caractère)] peut apporter une information plus fiable (Govindaraj et al., 2011). Les caractères (LAF, VP et NF) ont exprimé une héritabilité au sens large très élevée et ils ont exprimé un gain génétique attendu élevé aussi, ceci traduit l'action additive des gènes. (Johnson et al., 1955 ; Kashif et al., 2003, cités par Nerbéwendé et al.,2014).

par des faibles écarts entre les coefficients de variation phénotypique et génotypique, et une forte héritabilité au sens large pour tous les caractères étudiés. Le gain génétique attendu élevé exprimé par les caractères (LAF, $\mathrm{VP}$ et NF) et un gain génétique attendu moyen exprimé par le poids de mille grains (PMG) traduisent l'action additive des gènes dans l'expression des caractères. Ces caractères peuvent donc directement intervenir dans l'amélioration par sélection. Cette étude mérite d'être complétée par une caractérisation moléculaire et une analyse biochimique des grains.

genetic advance in Phenomorphic and agronomic traits of Tef [Eragrostis tef (Zucc.) 
Trotter] germplasm from eight Regions of Ethiopia. Hereditas 134, no. 2 : 103-113

Ayana A. et Bekele E., 2000. Geographical patterns of morphological variation in sorghum (Sorghum bicolor (L.) Moench) germplasm from Ethiopia and Eritrea : Quantitative Characters. Euphytica, 115: 91 - 104.

Burton G.W. and Devane E.M., 1953. Estimating heritability in tall fescue (Festuca arundinacea) from replicated clonal material. Agronomy Journal, 45, 478-481.

Djè Y., Heuertz M., Ater M., Lefebvre C. \& Vekemans X., 2007. Évaluation de la diversité morphologique des variétés traditionnelles de sorgho du Nordouest du Maroc. Biotechnol. Agron. Soc. Environ., 11 (1) : 39-46.

Doss S. G., Chakraborti S. P., Roychowdhuri S., Das N. K., Vijayan K., Ghosh P. D., Rajan M. V. et Qadri S. M. H., 2012. Variability, heritability and genetic advance in mulberry (Morus spp.) for growth and yield attributes. Agricultural Sciences Vol.3, No.2, 208-213.

Drabo I., Zangre G. R., Sawadogo M. et Ouedraogo M., 2013. Genetic Variability and Estimates of Genetic Parameters in Burkina Faso's Pearl Millet Landraces. International Journal of Agriculture and Forestry 3(7) : 367-373.

Govindaraj M., Selvi B., Rajarathinam S. et Sumathi P., 2011. Genetic Variability and Heritability of Grain Yield Components and Grain Mineral Concentration in India's Pearl Millet (Pennisetum Glaucum (L) R. Br.) Accessions. African Journal of Food, Agriculture, Nutrition and Development 11, no. 3.

Hosseini S. J., Sarvestani Z. T., Pirdashti H., Afkhami A. et Hazrati S., 2012. Estimation of heritability and genetic advance for screening some rice genotypes at salt stress conditions. International journal of Agronomy and Plant Production. Vol., 3 (11), 475482.

Johnson H. W., H. F. Robinson et Comstock, R. E., 1955. Estimates of genetic and environmental variability in Soybeans. Agronomy Journal 47, no. 7: 314-318.

Kashif M., Ahmad J., Chowdhry MA., Perveen K., 2003. Study of Genetic Architecture of Some Important Agronomic Traits in Durum Wheat (Triticum durum). Asian Journal of Plant Sciences, 2 (9) : 708-712.

Kenga R., et Djorowe G., 2008. Variabilité morphologique des sorghos de saison sèche [Muskwari] du
Nord Cameroun. Plant Genetic Ressources Newsletter, 2008, No. 153 : 9-14.

Nebié B., 2014. Diversité génétique d'une collection de sorgho a tige sucrée [Sorghum bicolor (L.) Moench] du Burkina Faso. Thèse Unique, Université de Ouagadougou (Burkina Faso) $118 p$.

Nebié B., Gapili N., Traoré R. E., Nanema K R, Bationo/Kando P., Sawadogo M. \& Zongo J. D., 2012. Diversité phénotypique des sorghos à grains sucrés du centre nord du Burkina Faso. Sciences et techniques, sciences naturelles et agronomie vol 32, $\mathrm{N}^{\circ} 1$ et 2, 2010-2012.

Nerbéwendé S., Romaric K. N., Pauline B. K., Renan E. T., Baloua N., Djakaridia T., Mahamadou S., Jean-Didier Z., 2014. Évaluation de la diversité génétique des sorghos à grains sucrés (Sorghum bicolor (L.) Moench) du Nord du Burkina Faso. Journal of Applied Biosciences 84 :7654- 7664. ISSN 1997-5902.

Peter Visscher M., William G. H., et Naomi R. W., 2008. Heritability in the genomics Era-concepts and misconceptions, Nature Reviews Genetics 9, no. 4: 255-266.

Rex B., 2002. Breeding for quantitative traits in plants. Stemma press Woodbury Singh R. K.

Stanfield W. D., 1975. Génétics. Mc Graw-Hilllnc, New York, $281 \mathrm{p}$.

Sumathi P., Sumanth M. et Veerabadhiran P., 2010. Genetic variability for different biometrical traits In Pearl Millet genotypes (Pennisetum Glaucum LR BR.)," Electronic journal of plant breeding 1 , no. 4: 437-440.

USDA, World Agricultural Production, novembre 2016.

Vetriventhan M. et Nirmalakumari A., 2007. Studies on Variability Parameters in Pearl Millet (Pennisetum Glaucum (L.) R. Br.). Madras Agricultural Journal 94, no. 1/6:118-120.

Yakouba O., Abdoul-Aziz S., Ali M., Félix W. Z., et Oberline F. Y., 2017. Perception paysanne des perturbations pluviométriques et stratégies d'adaptation dans les systèmes de culture à sorgho repiqué en zone soudano-sahélienne du Cameroun. Afrique SCIENCE 13(4) (2017) 50 65 ISSN 1813-548X, http://www.afriquescience.info. 